\title{
Mycorrhizal fungi as a biocontrol agent.
}

\begin{abstract}
Arbuscular mycorrhizae fungi (AMF) are the symbiotic fungi that predominate in the roots and soils of agricultural crop plants. The AMF form beneficial symbioses in most terrestrial ecosystems and crop production systems. Ninty percent of land plant species are colonized by one or more of the mycorrhizal fungi species ranging from flowering to non flowering plants, while only a few plant families do not form this association. The relationship between mycorrhiza and plant is very widely spread among terrestrial vascular plants. The AMF must have a host to complete its life cycle and this association has been found to be mutually beneficial; thus, the fungus assists the plant in mineral nutrients uptake, while the plant supplies the fungus with carbon as a result of this relation. The negative-antagonistic interaction of AMF with various soilborne plant pathogens is the reason for their use as a biocontrol agents. Many workers have observed an antagonistic effect of AMF against some fungal pathogens.
\end{abstract}

Keyword: Biological control; Endo-mycorrhizal fungi; Disease; Control 